\title{
Risk factors of morbidity among children under age five in Ethiopia
}

\author{
Kasahun Takele ${ }^{1 *}$ (D) Temesgen Zewotir ${ }^{2}$ and Denis Ndanguza ${ }^{3}$
}

\begin{abstract}
Background: Childhood morbidities are a major cause of mortality of children in the developing countries particularly in Ethiopia. Regardless of the noticeable improvement in the reduction of under-five death in Ethiopia, childhood diarrhea and fever are still the leading cause of death. In Ethiopia, the burden of child mortality is alarming and calls for determined efforts in combating such health problems. Therefore, this study aimed to investigate the risk factors for childhood morbidity specifically for diarrhea and fever.

Methods: To gain insight into children's health issues, the 2016 Ethiopian Demographic and Health Survey data were used. Among the marginal models, alternating logistic regression that is an extension of the generalized estimating equation model was used to investigate the risk factors of childhood morbidity explicitly for diarrhea and fever.

Results: The results show that the child's sex, child's age, anemia level, husband education level, mother's work status, mother's marital status, breastfeeding status and region are all chosen significant risk factors related with childhood diarrhea disease and fever disease.

Conclusion: The study indicated that male children, 0-11 months aged children, 12-23 months aged children, anemic children, husband with a lower education, mothers paid employment, non-breastfed children, regions of Amhara, Afar, Dire Dawa, Benishangul, Gambela, Oromia, SNNPR, Somali and Tigray were significantly associated with higher odds of morbidity in Ethiopia. Therefore, there is a need for children morbidity interventions intended to improve child health outcomes in the country.
\end{abstract}

Keywords: EDHS, GEE, ALR, Diarrhea, Fever

\section{Background}

Childhood diseases are among the most serious health issues facing developing countries. In 2016, globally acute respiratory infections, diarrhea and malaria were the leading causes of children under five death particularly $8 \%$ of deaths among children under age five caused by diarrhea [1]. According to the UNICEF report in 2016, approximately 5.6 million children under age of five die every year, which is decreased from over 12 million in 1990. Most of these children (80\%) are from sub-Saharan African and Southern Asia. Regardless of the considerable improvement in reducing childhood death, childhood survival persists a critical concern. Globally, about $75 \%$ of the under-five deaths are still caused by a handful of

\footnotetext{
* Correspondence: kastake10@gmail.com

${ }^{1}$ African Center of Excellence in Data Science, University of Rwanda, Kigali, Rwanda

Full list of author information is available at the end of the article
}

conditions like diarrhea, fever, cough and malaria. Furthermore, it is insupportable that 15,000 children die daily, commonly from preventable and treatable diseases such as diarrhea, malaria and fever [2]. Some of previous studies revealed that as diarrhea and fever are among the prevalent diseases that contributes to the burden of childhood morbidity and mortality [3]. Separately from its serious influence on child mortality, diarrhea and fever can result in long-term health effects, including depletion of immune strength, malnutrition and making children susceptible to other diseases $[4,5]$.

Numerous investigations in Africa revealed that infectious diseases as the leading causes of under age of five children death [6-9]. In Ethiopia, childhood morbidity and mortality remain high due to the burden associated with highly prevalent diseases such as diarrhea, fever, cough, malaria and HIV-AIDS. For instance, diarrhea contributes to more than one in every ten $(13 \%)$ child deaths in 
Ethiopia [10]. Furthermore, according to the 2016 Ethiopia Demographic and Health Survey report, 12\% of under-five children had a diarrheal episode and $14 \%$ of under-five children had a fever episode in the 2 weeks prior the survey [11]. This has encouraged medical researchers and statisticians to gain insight into this health problem with the view to developing strategies to combat it.

Likewise, some of studies have investigated the association between diseases and socioeconomic, demographic, environmental, and individual risk factors in Ethiopia and many other sub-Saharan African countries. Most of these investigations used logistic regression to identify the significant risk factors of diseases that associated to children morbidity [6, 12-15]. In addition, other articles focused only on diarrhea, using simple statistical analyses $[12,16,17]$. However, these previous studies on children diseases ignored association between children from the same household. As results, inference associated with the estimated parameters may not be correct means that the standard errors may be too small resulting in $p$-values that are too small and confidence intervals that are too narrow [18]. Furthermore, little is known and much work remains to be done to develop a better accepting approach that allows us to investigate childhood morbidities in a more accurate way.

The main objective of this work was to examine the effect of the socioeconomic, demographic, household and spatial characteristics related to children morbidity using the 2016 Ethiopia Demographic and Health Survey data. Identification of determinants of children diseases tends to assist to guide strategic planning, address strength and weakness of current policy, prioritize future research questions and interventions for addressing children morbidity and mortality problem in Ethiopia.

\section{Methods}

\section{Data source}

Ethiopia is a landlocked country located in East Africa, which categorized as sub-Saharan Africa. In this paper, we used the data set available from the 2016 Ethiopian DHS. This survey is the fourth cross-sectional investigation administered at household level. A stratified twostage cluster sampling procedure was used. Enumeration areas (EA) were the sampling units for the first stage and list of households in EA were the sampling unit in the second stage. The sample included 645 enumeration areas among 202 in urban areas and 443 in rural areas. The survey included questions designed to explore sociodemographic, socioeconomic, child health, maternal and environmental conditions at household level. The data was collected from women interviewees aged 15-49 years. In the survey, the health status of each interviewee's children aged less than 60 months in the 2016 survey was assessed by asking the interviewee 'Has your child had diarrhea, and fever in the last two weeks?' The occurrence of diarrhea and fever categorized as 'Yes' or 'No.' Overall, data on 8,742 'young' children was collected in the survey [11]. Table 1 provides information on categorical socioeconomic and demographic covariates, their categories, frequencies and association with diarrhea and fever. Childhood diarrhea defined as the frequent (three or more times per day) loss of liquid stools within 2 weeks period preceding to survey. Fever is an abnormally high body temperature, accompanied by shivering, headache, and restlessness [19]. The details on sampling methodology used in the survey can be found in [11].

\section{Marginal models}

Generalized Estimating Equation (GEE) and Alternating Logistic Regression (ALR) were used to model childhood morbidity, and compare the odds of a child being had diarrhea and /or fever given the various risk factors considered. Within these GEE and ALR models framework the households were considered to be clustered and not independent within each household. The response variables were the occurrence of diarrhea and/or fever (Yi: binominal variable, i.e., yes or no), and for this study we considered that the event occurred if the child had diarrhea and/or fever in the last 2 weeks of the survey. Consequently, the response variable was coded with 1 for child had diarrhea and/or fever and with 0 for child had no diarrhea and/or fever. The link function between the mean value $Y_{i}$ and the model covariates considered for GEE and working correlation structure is defined by:

$$
g\left(\omega_{i}\right)=\operatorname{logit}\left(\omega_{j}\right)=x_{i}^{\prime} \beta
$$

Where $g\left(\omega_{i}\right)$ is logit link function, $x_{i}$ is $n_{i} * x_{i}$ dimensional vector of known covariates, $\beta=(1 x p)$ dimensional vector of unknown parameters, $E\left(Y_{i}\right)=\omega_{i}$ is expected value of the response $Y_{i}$ in the cluster i which is binomially distributed as $y_{i} \sim \operatorname{bin}\left(n_{i}, \omega_{i}\right)$. In addition, GEE is nonlikelihood method that captures the association within households in terms of marginal correlations [18]. With this GEE model, the correlation structure of the data within each household was assumed to be of the independence, unstructured, exchangeable, and first order auto-regressive [20]. The parameter $\boldsymbol{\beta}$ are estimated by quasi-likelihood.

However, when the cluster sizes become larger, the simultaneous estimation of marginal mean and dependence structure can become computationally prohibitive using GEE. As a result, alternating logistic regression which measures pairwise association of two observations in the same household and follow the precision estimates for both the regression $(\boldsymbol{\beta})$ and the association $(\boldsymbol{\alpha})$ parameters considered $[18,21]$. Furthermore, unlike GEE, no 
Table 1 Distribution of childhood morbidity and its associated selected risk factors

\begin{tabular}{|c|c|c|c|c|c|c|}
\hline \multirow[t]{2}{*}{ Covariates } & \multicolumn{3}{|c|}{ Diarrhea recently ( $\mathrm{N}$ and \%) } & \multicolumn{3}{|c|}{ Fever recently ( $\mathrm{N}$ and \%) } \\
\hline & No & Yes & $p$-value & No & Yes & $p$-value \\
\hline \multicolumn{7}{|l|}{ Current age of child } \\
\hline 0-11 months & 1604(86.1) & 260(13.9) & 0.000 & $299(16.0)$ & $299(16.0)$ & 0.000 \\
\hline $12-23$ months & $1449(82.1)$ & $316(17.9)$ & & $1421(80.5)$ & $344(19.5)$ & \\
\hline 24-59 months & 4672(91.4) & $441(8.6)$ & & $4499(88.0)$ & $614(12.0)$ & \\
\hline \multicolumn{7}{|l|}{ Sex of child } \\
\hline Male & $3912(87.8)$ & $542(12.2)$ & 0.012 & $3806(85.5)$ & $648(14.5)$ & 0.654 \\
\hline Female & $3813(88.9)$ & $475(11.1)$ & & $3679(85.8)$ & 609 (14.2) & \\
\hline \multicolumn{7}{|l|}{ Anemia level } \\
\hline Anemic & $7517(88.2)$ & $1003(11.8)$ & 0.012 & $7283(85.5)$ & $1237(14.5)$ & 0.021 \\
\hline Not anemic & $208(93.7)$ & $14(6.3)$ & & $202(91.0)$ & $20(9.0)$ & \\
\hline \multicolumn{7}{|c|}{ Husband education level } \\
\hline No education & 3995 (89.7) & $457(10.3)$ & 0.000 & $3858(86.7)$ & $594(13.3)$ & 0.000 \\
\hline Primary & $2389(86.7)$ & $367(13.3)$ & & $2298(83.4)$ & $458(16.6)$ & \\
\hline Secondary & $776(86.5)$ & $121(13.5)$ & & $763(85.1)$ & $134(14.9)$ & \\
\hline Higher & $565(88.7)$ & $72(11.3)$ & & $566(88.9)$ & $71(11.1)$ & \\
\hline \multicolumn{7}{|l|}{ Currently working } \\
\hline No & 5609 (88.9) & $703(11.1)$ & 0.020 & $5453(86.4)$ & 859 (13.6) & 0.001 \\
\hline Yes & $2116(87.1)$ & $314(12.9)$ & & 2032 (83.6) & $398(16.4)$ & \\
\hline \multicolumn{7}{|l|}{ Marital status } \\
\hline Never in union & $51(91.1)$ & $5(8.9)$ & 0.696 & $42(75.0)$ & $14(25.0)$ & 0.002 \\
\hline Married & $7300(88.4)$ & $960(11.6)$ & & 7089 (85.8) & $1171(14.2)$ & \\
\hline Widowed & $94(90.4)$ & $10(9.6)$ & & $95(91.3)$ & $9(8.7)$ & \\
\hline Separated & $280(87.0)$ & $42(13.0)$ & & $259(80.4)$ & $63(19.6)$ & \\
\hline \multicolumn{7}{|c|}{ Breastfeeding status } \\
\hline No & 2589 (89.7) & $298(10.3)$ & 0.007 & 2470 (85.6) & $417(14.4)$ & 0.015 \\
\hline Yes & $5136(87.7)$ & $719(12.3)$ & & $5015(85.7)$ & $840(14.3)$ & \\
\hline
\end{tabular}

working assumptions about the third order and fourthorder odds ratios are required. Alternating logistic regression measures the association using the odds ratio, which is interpretable and more applicable for binary data $[18,21]$. Let $\gamma_{i j k}$ be the log odds ratio between outcomes $y_{i j}$ and $y_{i k}$, let $\mu_{i j}=p\left(y_{i j=1}\right)$ and $v_{i j k}=p\left(y_{i j}=1, y_{i k}=1\right)$, then the association of the two responses is defined as [22]:

$$
\begin{aligned}
& \operatorname{logit} p\left(Y_{i j}=1 / Y_{i k}=y_{i k}\right) \\
& \quad=\gamma_{i j k} Y_{i k}+\log \left(\frac{\mu_{i j}-v_{i j k}}{1-\mu_{i j}-\mu_{i k}+\mu_{i j k}}\right)
\end{aligned}
$$

Similar to GEE, the parameter $\boldsymbol{\beta}$ are estimated by quasi-likelihood. Descriptive statistics and chi-squared tests were carried out to identify associations between risk factors and outcome variables (diarrhea and fever) using version 21 of SPSS. P-values of less than 0.05 were considered statistically significant. Generalized estimating equation and alternating logistic regression models fit for this study were carried out with the SAS 9.4 version.

\section{Model selection}

The purpose of building a statistical model is to find an optimal model characterized by principles of generalizability, goodness-of-fit and parsimony based on model selection criteria. In this study, three working correlation assumptions exchangeable, first-order autoregressive, AR (1), and independence were used to select the best model with minimum qausi information criteria (QIC) and to select a working correlation structure [23]. In order to select important covariates related to diarrhea and fever backward selection method was used. With this method, we started with a full model containing all main effects and interactions to a more parsimonious model. 


\section{Results}

\section{Exploratory data analysis}

Figure 1 shows the prevalence of diarrhea by region in Ethiopia. It reveals that diarrhea disease (Yes) of children in the country is highly prevalent in Oromia, SNNPR and Tigray followed by Amhara, Afar, Gambela and Somali regions compared to Addis Ababa city. Likewise, the bar chart of Fig. 2 shows that fever disease (Yes) of children in the country is highly prevalent in Oromia, SNNPR and Tigray followed by Afar region compared to Addis Ababa city.

Besides, the results of cross-tabulation analysis for children morbidity are summarized in Table 1 . Crosstabulation analysis indicates that current age of child, anemia level, husband education level and breastfeeding status associated with childhood diarrheal disease and fever disease at a 5\% level of significance. Similarly, sex of child and marital status associated with childhood diarrheal disease and fever disease at a $5 \%$ level of significance, respectively. Table 1 indicates that higher prevalence of both diseases among children of age group between 12 to 23 months, $17.9 \%$ diarrhea and 19.5\% fever (diarrhea, $p$ value $<0.000$, fever, $p$-value $<0.000)$.

Moreover, from Table 1, the male children are more likely to have diarrhea compared to female counterpart $12.2 \%$ ( $p$-value $=0.012$ ). The children from mother paid employment are indicates higher prevalence rate of diarrhea and fever diseases than children from mother unemployed $(12.9 \%, p$-value $=0.020,16.4 \%, p$-value $<0.001)$ respectively. From the same table, it is indicated that anemic children are more vulnerable to diarrhea and fever 11.8 and $14.5 \%$ respectively than non-anemic children (diarrhea, $p$-value $=0.012$, fever, $p$-value $<0.021)$. Furthermore, breastfeeding is associated with diarrheal disease and fever disease (diarrhea, $p$-value $<0.000$, fever, $p$-value $<0.001)$. Table 1 shows that non-breastfed children have a lower rate of diarrhea. In addition, breastfed children have a lower rate of fever compared to non-breastfed children (14.3 vs. $14.4 \%)$. The education level of the father is highly associated with childhood morbidity (diarrhea, $p$-value $<0.000$, fever, $p$-value $<0.000)$.

\section{Results from marginal models}

Model with diarrhea as outcome and child sex, child age, anemia, husband education level, mother's work status and region as predictors were found to be the most parsimonious model. This model has the smallest QIC value for exchange, independence and unstructured working correlation structures. Lastly, exchangeable working correlation assumption was found to be more plausible than the other working correlation assumptions based on comparison of empirical and model based standard errors for the parameter estimates since the two standard errors were very close. Finally, using selected predictors, alternative logistic regression model which provides information about pairwise association of observations among two households within the same cluster was fitted. For diarrhea as outcome variable, the QIC values 6126.9290 and 6126.8773 for GEE and ALR models respectively, with parameter estimates, their corresponding empirically corrected standard errors and $p$-values presented in Table 2. The QIC for GEE and ALR are almost equal. However, empirically corrected standard errors under ALR are somewhat less than those of GEE and the small

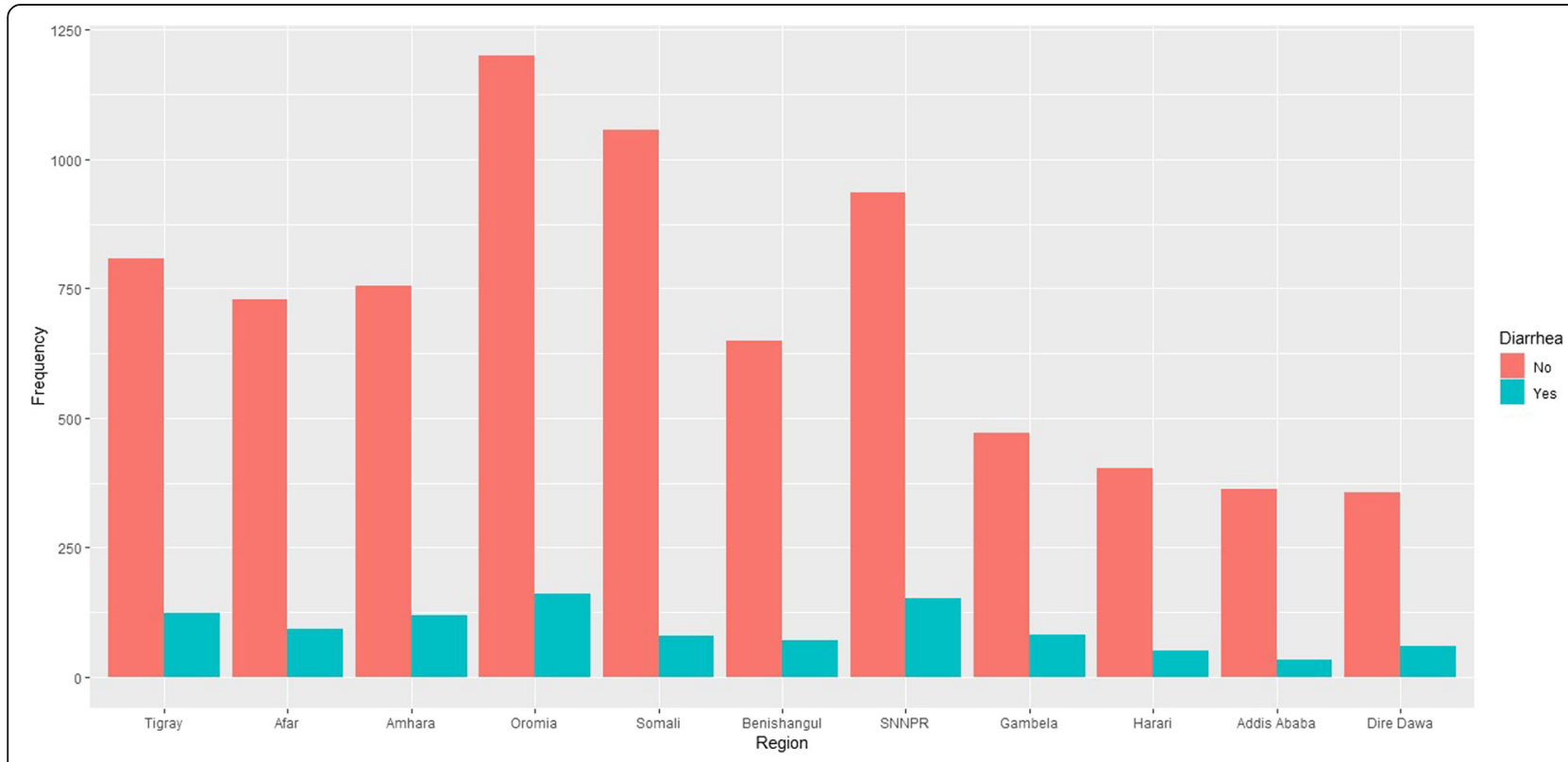

Fig. 1 Bar chart of childhood diarrhea morbidity by region 


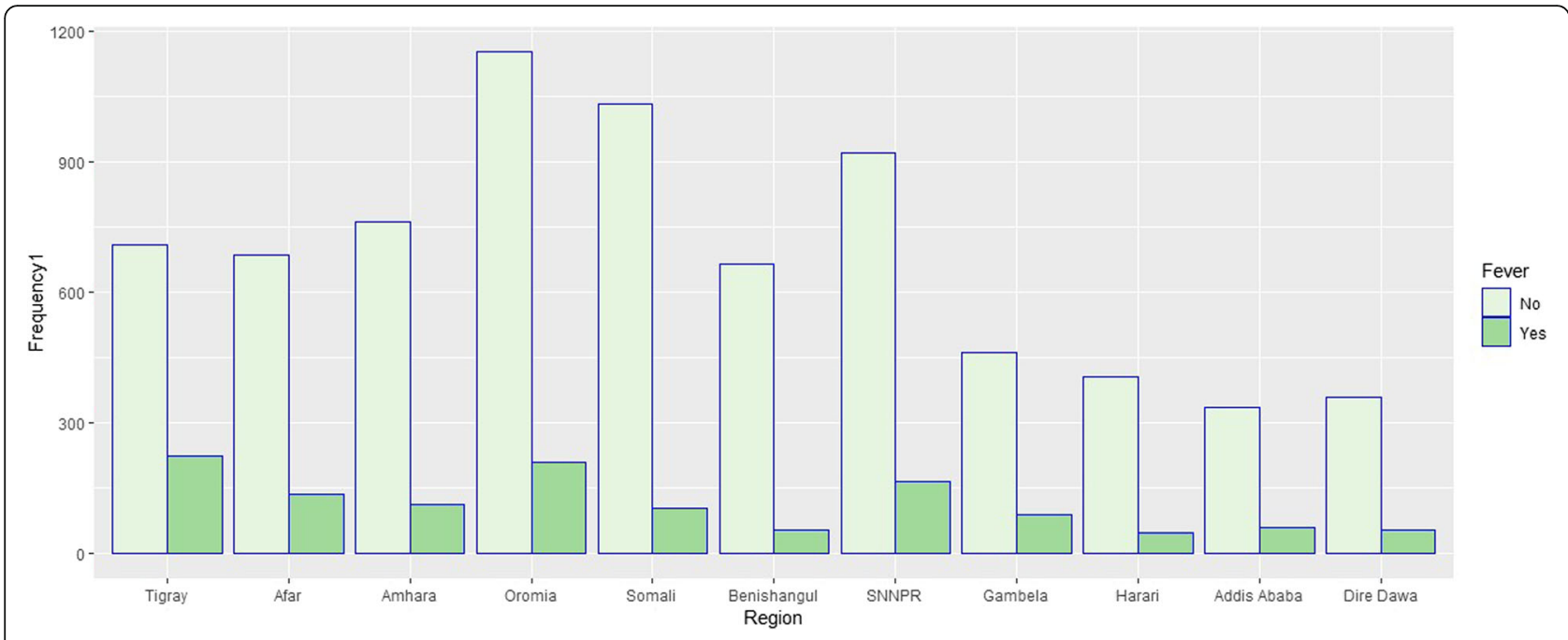

Fig. 2 Bar chart of childhood fever morbidity by region

Table 2 Parameter estimates and empirical standard errors of GEE and ALR models for Diarrhea

\begin{tabular}{|c|c|c|c|c|c|}
\hline \multirow{2}{*}{$\begin{array}{l}\text { Marginal Model } \\
\text { Effect }\end{array}$} & \multicolumn{3}{|c|}{ GEE } & \multicolumn{2}{|l|}{ ALR } \\
\hline & par & Est(s.e) & $p$-value & Est(s.e) & $P$-value \\
\hline Intercept & $\beta_{0}$ & $-3.3256(0.3445)$ & $<.0001$ & $-3.3279(0.3199)$ & $<.0001$ \\
\hline \multicolumn{6}{|c|}{ Current age of child (24-59 months $=$ ref) } \\
\hline 0-11 months & $\beta_{1}$ & $0.5518(0.0843)$ & $<.0001$ & $0.5529(0.0840)$ & $<.0001$ \\
\hline $12-23$ months & $\beta_{2}$ & $0.8384(0.0801)$ & $<.0001$ & $0.8402(0.0780)$ & $<.0001$ \\
\hline \multicolumn{6}{|c|}{ Sex of child (Male $=$ ref $)$} \\
\hline Female & $\beta_{3}$ & $-0.1327(0.0638)$ & 0.0376 & $-0.1332(0.0637)$ & 0.0366 \\
\hline \multicolumn{6}{|c|}{ Anemia level (not anemic $=$ ref.) } \\
\hline Anemic & $\beta_{4}$ & $0.5489(0.2641)$ & 0.0377 & $0.5507(0.2639)$ & 0.0369 \\
\hline \multicolumn{6}{|c|}{ Husband education level (No education $=$ ref) } \\
\hline Higher & $\beta_{5}$ & $0.0556(0.1454)$ & 0.7019 & $0.0564(0.1451)$ & 0.6972 \\
\hline Primary & $\beta_{6}$ & $0.2434(0.0828)$ & 0.0033 & $-0.2440(0.0827)$ & 0.0032 \\
\hline Secondary & $\beta_{7}$ & $0.2894(0.1165)$ & 0.0130 & $-0.2905(0.1163)$ & 0.0125 \\
\hline \multicolumn{6}{|c|}{ Currently working $($ Yes $=$ ref $)$} \\
\hline No & $\beta_{8}$ & $-0.1980(0.0763)$ & 0.0095 & $-0.1979(0.0762)$ & 0.0094 \\
\hline \multicolumn{6}{|c|}{ Region (Addis Ababa = ref) } \\
\hline Afar & $\beta_{9}$ & $0.5600(0.2251)$ & 0.0129 & $0.5610(0.2249)$ & 0.0126 \\
\hline Amhara & $\beta_{10}$ & $0.7600(0.2136)$ & 0.0004 & $0.7617(0.2134)$ & 0.0004 \\
\hline Benishangul & $\beta_{11}$ & $0.2779(0.2267)$ & 0.2204 & $0.2779(0.2263)$ & 0.2195 \\
\hline Dire Dawa & $\beta_{12}$ & $0.7616(0.2256)$ & 0.0007 & $0.7629(0.2254)$ & 0.0007 \\
\hline Gambela & $\beta_{13}$ & $0.7260(0.2221)$ & 0.0011 & $0.7280(0.2218)$ & 0.0010 \\
\hline Harari & $\beta_{14}$ & $0.4263(0.2425)$ & 0.0788 & $0.4293(0.2420)$ & 0.0761 \\
\hline Oromia & $\beta_{15}$ & $0.5142(0.2086)$ & 0.0137 & $0.5144(0.2084)$ & 0.0136 \\
\hline SNNPR & $\beta_{16}$ & $0.6846(0.2099)$ & 0.0011 & $0.6860(0.2096)$ & 0.0011 \\
\hline Somali & $\beta_{17}$ & $0.0310(0.2226)$ & 0.8894 & $0.0318(0.2223)$ & 0.8863 \\
\hline Tigray & $\beta_{18}$ & $0.6167(0.2141)$ & 0.0040 & 0.6193(0.2136) & 0.0037 \\
\hline Correlation & $\rho$ & -0.003887364 & & & \\
\hline Alpha & a & & & $-0.0530(0.0297)$ & 0.0035 \\
\hline QIC & & 6126.9290 & & 6126.8773 & \\
\hline
\end{tabular}


differences in parameter estimates attributed to the fact that ALR considers the association, but GEE treats the association as a nuisance parameter.

As a result, ALR model was the better model in explaining the population average association among diarrhea and the selected predictor variables. The interpretation rely on the ALR model. In ALR model parameter reflects the effect of predictors on the log odds of probability of diarrhea controlling all the other predictors in the model. Then, the odds ratio of variable can computed as $\left(\mathrm{OR}=e^{\beta_{j}}\right)$. The analysis under ALR suggests that child age is significantly associated to diarrhea disease and it was revealed that children who are between age group 0-11 months had exp.(0.5529) = 1.738 times higher odds of had diarrhea than those whose age group between 24 and 59 months. Children whose age group between 12 and 23 months is exp. $(0.8402)=2.32$ time more odds of had diarrhea than whose age group between 24 and 59 months. This indicates that child diarrhea increased by $74 \%$ for children age group between 0 and 11 months when compared to children whose age group between 24 and 59 months. Furthermore, child diarrhea increased by $132 \%$ for children age group between 12 and 23 months as compared to children whose age group between 24 and 59 months.

From Table 2, it was indicated that a statistically significant relation between sex of child and diarrheal disease. The odds that female children had diarrheal disease is exp. $(-0.1332)=0.875$ times lower compared to male children with a diarrheal disease. Furthermore, the analysis suggests that anemia is significantly related to children diarrheal disease $(p$-value $=0.0369)$. This means that estimated odds of had diarrhea for anemic children is $74 \%$ more than the estimated odds for those with non-anemic children. With respect to mothers paid employment effect, it was indicated that mothers paid employment is significantly related to child diarrheal disease. This indicates that, the estimated odds of diarrhea for children belong to mothers unemployed was exp. $(-0.1979)=0.82$ times lower than the estimated odds for mothers paid employment.

In addition, it was revealed that, the estimated odds of had diarrhea for children from regions of Afar, Amhara, Dire Dawa, Gambela, Oromia, SNNPR and Tigray are respectively $75,114,32,115,107,67,99$ and $86 \%$ more than the estimated odds of children from the central region (Addis Ababa). Furthermore, Table 2 presents that the estimated constant log odds ratios, which provides information about association between households within the same cluster. The estimated pairwise odds ratio relating two responses from the different households was found to have a small negative value $(-0.0530)$, this underlining the weak association in diarrheal disease between households.

\section{Fever}

For fever as an outcome variable, the model with child age, mother marital status, anemia level, breastfeeding status, husband education level, mother work status and region as predictor were found to be the best-fit model with the smallest QIC value. The QIC values 6996.7587 and 6996.6577 for GEE and ALR models respectively, with parameter estimates, their corresponding empirically corrected standard errors and $p$-values presented in Table 3. The QIC for GEE and ALR are almost the same. However, empirically corrected standard errors under ALR are somewhat less than those of GEE and the small differences in parameter estimates attributed to the fact that ALR considers the association unlike the GEE treat the association as a nuisance parameter. Consequently, ALR model was the better model in explaining the population average association among fever and the selected predictor variables. From Table 3, the analysis under ALR specifies that child age is significantly related to fever disease and it was observed that children who were between age group $0-11$ months had exp. $(0.4446)=1.556$ times higher odds of had fever than those whose age group 24-59 months. Children whose age group between 12 and 23 months had exp. $(0.6386)=1.994$ time more odds of children had fever than whose age group between 24 and 59 months. This indicates child fever increased by $56 \%$ for children age group 0-11 months when compared to children with had no fever. Mother marital status is the other important covariate that has a statistically significant relation with child fever disease. The odds of child from mother never in union is exp. $(1.1817)=3.289$ times higher compared to child from married mother. It was observed that child from separated mother had exp. $(0.8494)=2.338$ times more odds of had fever than child from married mother. This indicates that child from separated and never in union mother are associated with fever disease. With respect to husband education level, it was indicated that husband education level has statistically significantly relation with child fever disease. Higher education level of the husband was associated with 0.747 times less odds of fever in a child compared to husbands with a lower education (25\% lower odds). Furthermore, the analysis suggests that breast-feeding status is associated to children fever disease. This means that estimated odds of children had fever for breast-feeding is $80 \%$ less than the estimated odds for those with not currently breastfeeding children. As it can be shown from Table 3, mothers work status is significantly related to child fever disease. This indicates that, the estimated odds of fever for children belong to unemployed mother was exp. $(-0.2352)=0.79$ times lower than the estimated odds for mother paid employment. Furthermore, region statistically related to children fever disease. The estimated odds of had fever for children from Benishangul, Somali and Tigray 
Table 3 Parameter estimates and empirical standard errors of GEE and ALR models for Fever

\begin{tabular}{|c|c|c|c|c|c|}
\hline \multirow{2}{*}{$\begin{array}{l}\text { Marginal Model } \\
\text { Effect }\end{array}$} & \multicolumn{3}{|c|}{ GEE } & \multicolumn{2}{|l|}{ ALR } \\
\hline & par & Est(s.e) & $p$-value & Est(s.e) & $P$-value \\
\hline Intercept & $\beta_{0}$ & $-2.719(0.4666)$ & $<.0001$ & $-2.7160(0.4663)$ & $<.0001$ \\
\hline \multicolumn{6}{|c|}{ Current age of child (24-59 months $=$ ref) } \\
\hline 0-11 months & $\beta_{1}$ & $0.4446(0.0898)$ & $<.0001$ & $0.4446(0.0898)$ & $<.0001$ \\
\hline $12-23$ months & $\beta_{2}$ & $0.6384(0.0794)$ & $<.0001$ & $0.6386(0.0794)$ & $<.0001$ \\
\hline \multicolumn{6}{|l|}{ Marital status } \\
\hline Married & $\beta_{3}$ & $0.5706(0.3758)$ & 0.1289 & $0.5694(0.3756)$ & 0.1295 \\
\hline Never in union & $\beta_{4}$ & $1.1839(0.4765)$ & 0.0130 & $1.1817(0.4764)$ & 0.0131 \\
\hline Separated & $\beta_{5}$ & $0.8503(0.3988)$ & 0.0330 & $0.8494(0.3987)$ & 0.0331 \\
\hline \multicolumn{6}{|c|}{ Anemia level (Not anemic = ref.) } \\
\hline Anemic & $\beta_{6}$ & $0.3780(0.2415)$ & 0.0175 & $0.3760(0.2411)$ & 0.1188 \\
\hline \multicolumn{6}{|c|}{ Breastfeeding status (no = ref) } \\
\hline Yes & & $-0.2214(0.0717)$ & 0.0020 & $-0.2215(0.0717)$ & 0.002 \\
\hline \multicolumn{6}{|c|}{ Husband education level (No education = ref) } \\
\hline Higher & $\beta_{7}$ & $-0.2902(0.1428)$ & 0.0421 & $-0.2904(0.1428)$ & 0.0420 \\
\hline Primary & $\beta_{8}$ & $0.2457(0.0729)$ & 0.0007 & $0.2459(0.0729)$ & 0.0007 \\
\hline Secondary & $\beta_{9}$ & $0.1076(0.1077)$ & 0.3181 & $0.1075(0.1077)$ & 0.3182 \\
\hline \multicolumn{6}{|c|}{ Currently working (Yes = ref) } \\
\hline No & $\beta_{10}$ & $-0.2536(0.0702)$ & 0.0003 & $-0.2539(0.0702)$ & 0.0003 \\
\hline \multicolumn{6}{|c|}{ Region (Addis Ababa = ref) } \\
\hline Afar & $\beta_{11}$ & $0.2352(0.1833)$ & 0.1993 & $0.2352(0.1833)$ & 0.1993 \\
\hline Amhara & $\beta_{12}$ & $-0.0868(0.1898)$ & 0.6474 & $-0.0872(0.1898)$ & 0.6460 \\
\hline Benishangul & $\beta_{13}$ & $-0.7778(0.2109)$ & 0.0002 & $-0.7791(0.2110)$ & 0.0002 \\
\hline Dire Dawa & $\beta_{14}$ & $-0.1015(0.2106)$ & 0.6298 & $-0.1013(0.2106)$ & 0.6304 \\
\hline Gambela & $\beta_{15}$ & $0.1732(0.1914)$ & 0.3655 & $0.1730(0.1913)$ & 0.3660 \\
\hline Harari & $\beta_{16}$ & $-0.3806(0.2157)$ & 0.0776 & $-0.3816(0.2157)$ & 0.0770 \\
\hline Oromia & $\beta_{17}$ & $0.0417(0.1574)$ & 0.7909 & $0.0416(0.1574)$ & 0.7917 \\
\hline SNNPR & $\beta_{18}$ & $0.0175(0.1681)$ & 0.9172 & $0.0171(0.1681)$ & 0.9190 \\
\hline Somali & $\beta_{19}$ & $-0.4475(0.1849)$ & 0.0155 & $-0.4473(0.1849)$ & 0.0156 \\
\hline Tigray & $\beta_{20}$ & $0.5627(0.1687)$ & 0.001 & $0.5629(0.1687)$ & 0.0008 \\
\hline Correlation & $\rho$ & -0.002804291 & & & \\
\hline Alpha & $a$ & & & $-0.0204(0.0278)$ & 0.0046 \\
\hline QIC & & 6996.7587 & & 6996.6577 & \\
\hline
\end{tabular}

regions are respectively 46,64 and $86 \%$ less than the estimated odds of children from the central region (Addis Ababa) except for Tigray region. Table 3 presents that the estimated constant log odds ratios, which provides information about association between households within the same cluster. The estimated pairwise odds ratio relating two responses from the different households was found to have a small negative association (-0.0204), this indicates weak association between children in different households in terms of fever disease.

\section{Discussion}

This study utilized 2016 Ethiopian Demographic and Health Survey data to identify the risk factors associated with diarrhea and fever among children of under-five in Ethiopia. These indicators might be differently distributed within as well as across households, since each indicates a different mechanism by which such morbidity are acquired. The marginal models were employed to account for clustering. The study found an overall incidence of diarrhea and fever 12 and 14\% respectively. For diarrhea as a response, alternating logistic regression 
analysis revealed that the covariates: male children, $0-11$ months aged children, 12-23 months aged children, anemic children, husband with a lower education, mothers paid employment, Afar, Amhara, Dire Dawa, Gambela, Oromia, SNNPR and Tigray regions were significantly associated with higher odds of diarrhea. For fever as an outcome variable, $0-11$ months aged children, 12-23 months aged children, children of never in union mother, children of separated mother, non-breastfed children, husband with a lower education, mothers paid employment, Benishangul, Somali and Tigray were significantly associated with higher odds of fever. The result shows that the age of child positively associated with the child morbidity. Children whose age group between 12 and 23 months are more probable to had diarrhea and fever morbidity than whose age group between 24 and 59 months. Similarly, children whose age group between 0 and 11 months are more at high risk of diarrhea and fever morbidity compared to the reference group. This finding is in line with that of $[3,6,24]$. In addition, the results show that risk of diarrhea is lower among female children than among male children. This finding is consistent with that of $[3,11,24]$. This may be due to biologic reasons and gender discrimination. Children caught by anemia are at higher risk of diarrhea and fever morbidity as compared to non-anemic children. This result is in agreement with previous findings such as $[24,25]$ among others.

Furthermore, the study found that the husband education level is an important socioeconomic factor that affects the health status of children in Ethiopia. Children from lower educated father are more at high risk of diarrhea morbidity and fever morbidity than a father at least who attended primary school. The result suggests that children from mother who are currently working are more at high risk of diarrhea morbidity and fever morbidity than children from mother who are currently not working. This result is in agreement with previous findings such as [3].

Lastly, the results indicate that children from some of regions like Amhara, Afar, Dire Dawa, Gambela, Oromia, SNNPR and Tigray are at higher risk of diarrheal morbidity compared to children from Addis Ababa. Likewise, children from Tigray region are at higher risk of fever morbidity. The finding is in line with that of $[6,26]$.

\section{Conclusions}

In this paper, we studied socioeconomic, demographic, household and spatial determinants of children morbidity measured through prevalence of diarrheal and fever using Ethiopian Demographic and health Survey data. Our analyses show that marginal models are needed to adequately account for the correlation between responses of interest in subjects from the same cluster to identify the risk factors of childhood diarrhea and fever. With a traditional logistic regression model we cannot detect pairwise association of two observations in the same cluster. The alternating logistic regression analysis shows that the male children, 0-11 months aged children, 12-23 months aged children, anemic children, husband with a lower education, mothers paid employment, non-breastfed children, Amhara region, Afar region, Dire Dawa, Benishangul region, Gambela region, Oromia region, SNNPR region, Somali region and Tigray region were significantly associated with higher odds of morbidity in Ethiopia. The findings of this study have valuable implications to support strategies and interventions to address diarrhea and fever in children aged five or younger. For further study, we will focus on the geographic and socioeconomic determinants of childhood morbidity in Ethiopia.

\section{Abbreviations}

ALR: Alternating Logistic Regression; DHS: Demographic and health survey; EA: Enumeration area; EDHS: Ethiopia demographic and health survey; GEE: Generalized Estimating Equation; QIC: Qausi information criteria, firstorder autoregressive, AR (1); SNNP: Southern Nations Nationalities and Peoples Region

\section{Acknowledgements}

We thank, ORC marco, measure DHS for giving us access for the data file.

\section{Authors' contributions}

KT acquired the data, analyzed and prepared the draft manuscript. TZ, as a principal advisor for KT, advised and supervised the start, the analysis and the write-up of the manuscript. DND, advised and supervised the analysis and the write-up of the manuscript. All authors read and approved the final manuscript.

Funding

None.

Availability of data and materials

The data can be accessed from http: //www.dhsprogram.com/ by registering and requesting the datasets.

Ethics approval and consent to participate

Letter of consent was received from the Measure DHS International Program, which authorized the data sets. All the data that used in this study are publicly available and do not in any way allow respondents, households, or sample communities to be identified. Confidentiality of data maintained anonymously.

Consent for publication

Not applicable.

\section{Competing interests}

The authors declare that they have no competing interests.

\section{Author details}

${ }^{1}$ African Center of Excellence in Data Science, University of Rwanda, Kigali, Rwanda. ${ }^{2}$ School of Mathematics, Statistics and Computer Sciences, University of KwaZulu-Natal, Durban, South Africa. ${ }^{3}$ College of Science and Technology, University of Rwanda, Kigali, Rwanda.

Received: 18 January 2019 Accepted: 2 July 2019

Published online: 15 July 2019

\section{References}

1. World health statistics. Monitoring health for the SDGs, sustainable development goals. Geneva: World Health Organization; 2018.

2. Hug L, Sharrow D, You D. Levels and trends in child mortality: report 2017 Estimates developed by the UN Inter-agency Group for Child Mortality Estimation. 2017. 
3. Khatab K, Fahrmeir L. Analysis of childhood morbidity with geoadditive probit and latent variable model: a case study for Egypt. Am J Trop Med Hyg. 2009;81(1):116-28.

4. Kumi-Kyereme A, Amo-Adjei J. Household wealth, residential status and the incidence of diarrhea among children under-five years in Ghana. J Epidemiol Global Health. 2016;6(3):131-40.

5. Fuentes R, Pfütze T, Seck P. 2006/5 a logistic analysis of diarrhea incidence and access to water and sanitation; 2006.

6. Bogale GG, Gelaye KA, Degefie DT, Gelaw YA. Spatial patterns of childhood diarrhea in Ethiopia: data from Ethiopian demographic and health surveys (2000, 2005, and 2011). BMC Infect Dis. 2017;17(1):426.

7. Abhulimhen-lyoha BI, Okolo AA. Morbidity and mortality of childhood illnesses at the emergency paediatric unit of the University of Benin Teaching Hospital, Benin City. Niger J Paediatr. 2012;39(2):71-4.

8. Onyiriuka AN. Morbidity and mortality patterns of post-neonatal paediatric medical admissions in a large mission hospital in Benin City, Nigeria; 2005.

9. Ibeziako SN, Ibekwe RC. Pattern and outcome of admissions in the Children's emergency room of the University of Nigeria Teaching Hospital, Enugu. Niger J Paediatr. 2002;29(4):103-8.

10. World Health Organization. Global status report on noncommunicable diseases 2014. Geneva: World Health Organization; 2014.

11. CSACE I. Ethiopia Demographic and Health Survey 2016. Addis Ababa, and Rockville: CSA and ICF; 2016.

12. Woldu W, Bitew BD, Gizaw Z. Socioeconomic factors associated with diarrheal diseases among under-five children of the nomadic population in Northeast Ethiopia. Trop Med Health. 2016:44(1):40.

13. Zeleke AT, Alemu ZA. Determinants of under-five childhood diarrhea in Kotebe health center, Yeka Sub City, Addis Ababa, Ethiopia: a case control study. Global J Med Res. 2014;:14(4):9.

14. Regassa W, Lemma S. Assessment of diarrheal disease prevalence and associated risk factors in children of 6-59 months old at Adama District rural Kebeles, eastern Ethiopia, January/2015. Ethiop J Health Sci. 2016;26(6):581-8.

15. Arif A, Arif GM. Socio-Economic Determinants of Child Health in Pakistan. Acad Res Int. 2012;2(1):398.

16. OConnell BJ, Quinn MA, Scheuerman P. Risk factors of diarrheal disease among children in the east African countries of Burundi Rwanda and Tanzania. Global J Med Public Health. 2017;6(1):8.

17. Thiam S, Diène AN, Fuhrimann S, Winkler MS, Sy I, Ndione JA, Schindler C, Vounatsou P, Utzinger J, Faye O, Cissé G. Prevalence of diarrhoea and risk factors among children under five years old in Mbour, Senegal: a crosssectional study. Infect Dis Poverty. 2017;6(1):109.

18. Molenberghs $\mathrm{G}$, Verbeke $\mathrm{G}$. Models for discrete longitudinal data; 2005.

19. World Health Organization. Integrated Management of Childhood Illness (IMCI). 2010.

20. Bena J, Mclntyre S. Survival methods for correlated time-to-event data. In: MWSUG 2008 conference; 2008. p. 12-4.

21. Carey V, Zeger SL, Diggle P. Modelling multivariate binary data with alternating logistic regressions. Biometrika. 1993;80(3):517-26.

22. Liang KY, Zeger SL. Regression analysis for correlated data. Annu Rev Public Health. 1993;14(1):43-68.

23. Liang KY, Zeger SL. Longitudinal data analysis using generalized linear models. Biometrika. 1986;73(1):13-22.

24. Kandala NB, Ji C, Stallard N, Stranges S, Cappuccio FP. Spatial analysis of risk factors for childhood morbidity in Nigeria. Am J Trop Med Hyg 2007:77(4):770-9.

25. Habyarimana F, Zewotir T, Ramroop S. Structured additive Quantile regression for assessing the determinants of childhood Anemia in Rwanda. Int J Environ Res Public Health. 2017;14(6):652.

26. Alebel A, Tesema C, Temesgen B, Gebrie A, Petrucka P, Kibret GD. Prevalence and determinants of diarrhea among under-five children in Ethiopia: a systematic review and meta-analysis. PLoS One. 2018:13(6): e0199684.

\section{Publisher's Note}

Springer Nature remains neutral with regard to jurisdictional claims in published maps and institutional affiliations.

\section{Ready to submit your research? Choose BMC and benefit from}

- fast, convenient online submission

- thorough peer review by experienced researchers in your field

- rapid publication on acceptance

- support for research data, including large and complex data types

- gold Open Access which fosters wider collaboration and increased citations

- maximum visibility for your research: over $100 \mathrm{M}$ website views per year

At $\mathrm{BMC}$, research is always in progress.

Learn more biomedcentral.com/submissions 